\title{
THE JOURNAL OF PHYSICAL
}

Subscriber access provided by Caltech Library

C: Surfaces, Interfaces, Porous Materials, and Catalysis

Initial Decomposition of HMX Energetic Material from Quantum Molecular Dynamics and the Molecular Structure Transition of \#- to \#-HMX

Caichao Ye, Qi An, Wenqing Zhang, and William A. Goddard

J. Phys. Chem. C, Just Accepted Manuscript • Publication Date (Web): 26 Mar 2019

Downloaded from http://pubs.acs.org on March 26, 2019

\section{Just Accepted}

"Just Accepted" manuscripts have been peer-reviewed and accepted for publication. They are posted online prior to technical editing, formatting for publication and author proofing. The American Chemical Society provides "Just Accepted" as a service to the research community to expedite the dissemination of scientific material as soon as possible after acceptance. "Just Accepted" manuscripts appear in full in PDF format accompanied by an HTML abstract. "Just Accepted" manuscripts have been fully peer reviewed, but should not be considered the official version of record. They are citable by the Digital Object Identifier (DOI@). "Just Accepted" is an optional service offered to authors. Therefore, the "Just Accepted" Web site may not include all articles that will be published in the journal. After a manuscript is technically edited and formatted, it will be removed from the "Just Accepted" Web site and published as an ASAP article. Note that technical editing may introduce minor changes to the manuscript text and/or graphics which could affect content, and all legal disclaimers and ethical guidelines that apply to the journal pertain. ACS cannot be held responsible for errors or consequences arising from the use of information contained in these "Just Accepted" manuscripts. 


\title{
Initial Decomposition of HMX Energetic Material from Quantum Molecular Dynamics and the Molecular Structure Transition of $\beta$ - to $\delta$-HMX
}

\author{
Cai-Chao Ye 1, 2, *, Qi An 2, 3, Wen-Qing Zhang ${ }^{1}$ and William A. Goddard III 2, * \\ ${ }^{1}$ Academy for Advanced Interdisciplinary Studies and Department of Physics, Southern University of Science and \\ Technology (SUSTech), Shenzhen 518055, P. R. China \\ ${ }^{2}$ Materials and Process Simulation Center (MSC), California Institute of Technology, Pasadena, California 91125, \\ United States \\ ${ }^{3}$ Department of Chemical and Materials Engineering, University of Nevada-Reno, Reno, Nevada 89577, United States
}

\begin{abstract}
We demonstrate the use of quantum molecular dynamics to identify the $\beta$ - to $\delta$-molecular structure transition in bulk phase HMX, which has been considered as the main reason of the increased sensitivity in the thermal decomposition of HMX. Both physical and chemical changes accompany this transition, but no previous study has shown conclusively which specific change, or set of changes, is responsible. We find the initial decomposition mechanism of HMX can explain this issue sensitivity. Our DFT simulations of the periodic system followed by detailed finite cluster calculations of the transition states find two distinct initial unimolecular reaction pathways in $\beta$-HMX, that operate simultaneously. (1) For the HONO releasing reaction, $\beta$-HMX first transformed to an intermediate in which one parallel $\mathrm{N}-\mathrm{NO}_{2}$ group transitions from chair to boat with a low $+1.2 \mathrm{kcal} / \mathrm{mol}$ barrier, followed by unimolecular HONO release $(+42.8 \mathrm{kcal} / \mathrm{mol}$ barrier, RDS). (2) For the $\mathrm{NO}_{2}$ cleavage reaction, $\beta$-HMX first transforms to the $\delta$-HMX structure in two steps, with low barriers of +1.9 and $+7.6 \mathrm{kcal} / \mathrm{mol}$ for each step, followed by unimolecular $\mathrm{NO}_{2}$ release $(+31.3 \mathrm{kcal} / \mathrm{mol}$ barrier$)$. Starting with $\delta$-HMX, we find an initial unimolecular $\mathrm{NO}_{2}$ cleavage and then an independent HONO releasing reaction, with the barriers of $+31.6\left(\mathrm{NO}_{2}\right.$ cleavage) and $+38.9 \mathrm{kcal} / \mathrm{mol}$ (HONO releasing). We find the constant proportional simulated initial structure transition temperature is $453 \mathrm{~K}$, which is consistent with the experimental results $(466 \mathrm{~K})$.
\end{abstract}

\section{INTRODUCTION}

There remain many uncertainties about the dynamic behavior of energetic materials (EMs), such as HMX (octahydro-1,3,5,7-tetranitro-1,3,5,7-tetrazocine), is complicated by the range of structural phases exhibited by many EMs ${ }^{1}$. HMX is one such material used as a crystalline high explosive that shows a variety of behaviors when subjected to various thermal fields. HMX exists four polymorphs, labeled $\alpha-, \beta-, \gamma-$, and $\delta$-, with the crystal structures known for $\alpha, \beta$, and $\delta^{2,3}$. These different crystal types of HMX exhibit different 
physico-chemical and detonation properties, and each crystal type can be recrystallized from the appropriate solution at different cooling rates. The $\beta$ and $\delta$ forms are of particular interest because they are the polymorphs used in typical formulations of composites for various applications. $\beta$-HMX is a low temperature form, while $\delta$-HMX is a high temperature form. $\delta$ is observed as heating of $\beta$-HMX when the temperature between 165 $210{ }^{\circ} \mathrm{C} 4,5$. Brill et al. reported kinetic measurements of the $\beta$ - to $\delta$ - transition, showing that it is first order with an activation energy and frequency factor resemble the values attributed to chemical decomposition, supporting previous speculation that the initial decomposition step in $\beta$-HMX is coupled to, or preceded by, the formation of $\delta$-HMX ${ }^{2,5}$. Many in situ measurements of the transformation rate has been reported for HMX ignition experiments. Henson et al. used second harmonic generation (SHG) as a fast in situ probe of the $\beta-\delta$ transition in HMX, and first demonstrated the technique by monitoring the transition during slow heating ${ }^{1,6}$. Although several thermodynamics and kinetics models of HMX crystal transition have been reported ${ }^{6-9}$, the kinetics of the $\beta-\delta$ transition have not yet been incorporated into any decomposition mechanisms of HMX, because it is very difficult and hazardous to observe and track the decomposition progress of energetic materials, and because the observed products may be the result of several sequential steps.

Recently, we reported great progress in using quantum mechanics (QM) based reactive dynamics to predict the complicated, detailed reaction barriers and kinetics of bulk phase reaction mechanisms ${ }^{10}$, especially for energetic materials ${ }^{11-15}$, with detailed confirmation from experiment and concomitant interpretation of experiment, making it possible to determine the initial decomposition steps of HMX solid phase reactions .

In this work, we want to elucidate the initial thermal decomposition mechanisms of HMX, we report here DFT based molecular dynamics (MD) simulations, using the forces from the PBE-ulg ${ }^{16}$ flavor of DFT, to examine the initial decomposition of $\beta$ - and $\delta$-HMX. These studies on the initial reaction of HMX examine the atomistic details of the $\beta$ - to $\delta$ - molecular structure transition and the decomposition mechanisms, providing an enriched understanding the $\beta$ - to $\delta$-crystal transformation of HMX.

\section{METHODS}

\subsection{Simulation method}

In this work, we perform the DFT based MD simulations. The interatomic forces were calculated in the framework of DFT 17-18, where exchange and correlation were processed with the generalized gradient approximation (GGA), and we used the PBE functional form plus the $u l g$ corrections for London dispersion 16.

We used the VASP package ${ }^{19-22}$ to perform the periodic DFT calculations. For the crystalline structures optimization, we use a kinetic energy cut-off of $500 \mathrm{eV}$, and it gives excellent convergence in total energies, energy differences, and lattice parameters. For the DFT-MD calculations, we also use $500 \mathrm{eV}$ energy cut-offs 
and only the gamma point for the supercell calculations. For geometry optimization, we set a $1 \times 10^{-6} \mathrm{eV}$ energy difference and a $1 \times 10^{-3} \mathrm{eV} / \AA$ force as convergence criteria for solving the electronic wave function. For DFT-MD simulation, we set a $1 \times 10^{-5} \mathrm{eV}$ energy difference and a $1 \times 10^{-3} \mathrm{eV} / \AA$ force as convergence criteria for solving the electronic wave function.
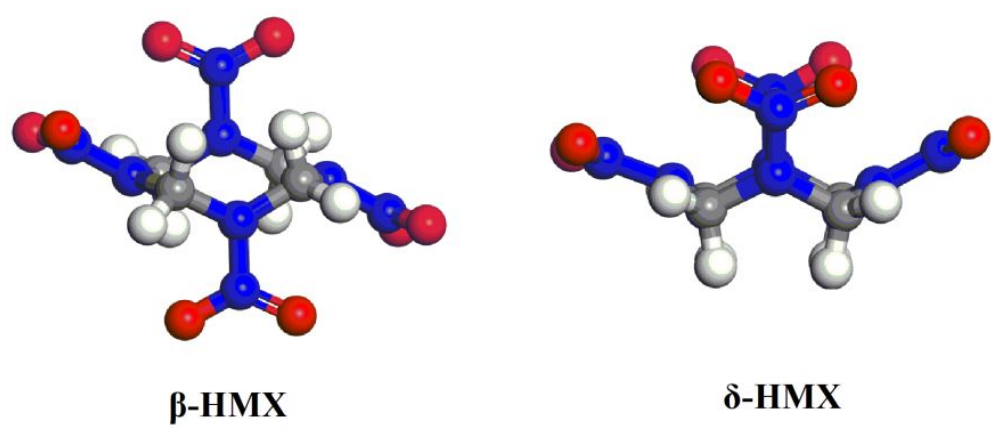

Figure 1 Molecular structures of $\beta$-HMX and $\delta$-HMX.

Two HMX crystalline phases ( $\beta$-HMX and $\delta$-HMX) were used in the DFT-MD simulations, We get the initial unit cell from CCSD database, and the $\beta$-HMX unit cell structure and $\delta$-HMX unit cell structure can be find in the Supporting Information. For $\beta$-HMX, the MD use a periodic cell which have 8 molecules (224 atoms per cell), obtained by replicating the unit cell twice along the " $a$ " and " $b$ " directions; For $\delta$-HMX, the MD considered 6 molecules per unit cell. Then before doing the molecule dynamics simulations, we optimized the two structures individually. The two optimized initial structures of $\beta$-HMX and $\delta$-HMX for MD simulations are shown in Figure S1.

Then we performed the DFT-MD cook-off simulations: First we heated the optimized initial systems 20 $\mathrm{K}$ to $300 \mathrm{~K}$ in $2 \mathrm{ps}$. Then the system was equilibrated at $300 \mathrm{~K}$ for $1 \mathrm{ps}$ using the NVT (constant volume, constant temperature and constant number of atoms) ensemble, with the Nose-Hoover thermostat and time constant set to $0.1 \mathrm{ps}$. Finally, the system was heated from $300 \mathrm{~K}$ to $3000 \mathrm{~K}$ in $20 \mathrm{ps}$ (heating rate $\sim 135 \mathrm{~K} / \mathrm{ps}$ ), and the volume was fixed. A time step of $1 \mathrm{fs}$ was used to integrate the equations of motion. To analyse the fragments in the DFT-MD simulation, a bond length cut-off of 1.5 times of the normal ones were used (see Table S1).

\subsection{Reaction mechanism by finite cluster calculation}

To examine the detail reaction mechanisms which discovered during the periodic DFT-MD simulations, we extracted the reaction involved molecule structures from the MD trajectories, and using the Jaguar program ${ }^{23}$ performed the gas phase reactions locate the nearby transition state (TS), at the level of $6-311++\mathrm{G}^{* *}$. We validated the TSs by only have one negative eigenvalue for the Hessian. And then we do the intrinsic reaction coordinate (IRC) scans, to connect the TS to nearby reactant and the product structures ${ }^{24}$. In order to get the free energies, we obtained the vibrational frequencies by diagonalized the mass reduced Hessian. And the thermodynamic properties were evaluated at $298.15 \mathrm{~K}$ and $1 \mathrm{~atm}$. 


\section{RESULTS \& DISCUSSION}

From our DFT-MD simulations, we obtained a detailed, molecular-level reaction pathway of the thermal decomposition in condensed phase $\beta$ - and $\delta$ - HMX. We can extract valuable information about complicated chemical progress involved ${ }^{25}$. In this work, we studied thermal decomposition of condensed phase $\beta$ - and $\delta$ HMX crystals, and focus on examining the initial reaction pathways to investigate the $\beta$ - to $\delta$ - molecular structure transition before the initial reaction occurs. As phase transition requires the cell change in the simulation, we didn't study in this work.

\subsection{Reaction mechanisms from DFT-MD}

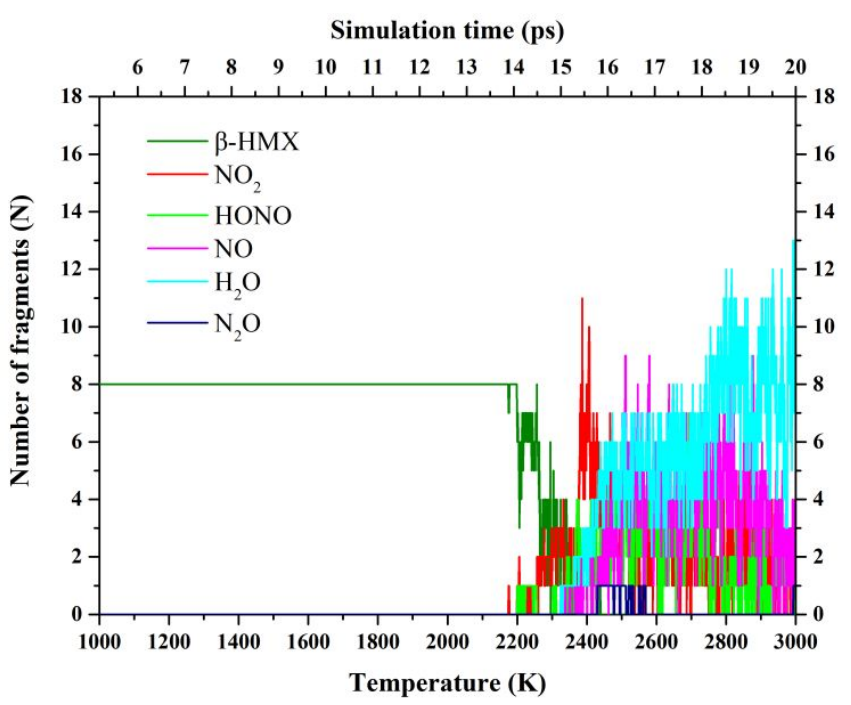

(a)

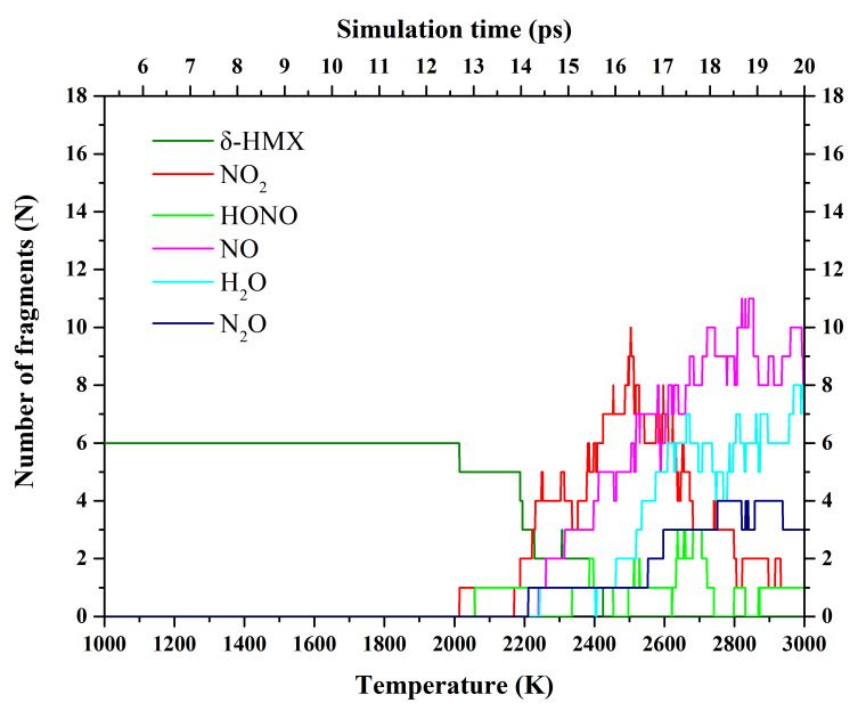

(b)

Figure 2 (a) Species analysis for $\beta$-HMX cookoff DFT-MD simulation (heating from 300 to $3000 \mathrm{~K}$ in $20 \mathrm{ps}$ ). $\beta$-HMX first decomposed at 2200 2250 K ( 14 ps), first $\mathrm{HONO}$ and $\mathrm{NO}_{2}$ molecules releasing almost at the same time. (b) Species analysis for $\delta$-HMX cookoff DFT-MD simulation (heating from 300 to $3000 \mathrm{~K}$ in $20 \mathrm{ps}$ ). $\delta$-HMX first decomposed at $\sim 2010 \mathrm{~K}(12.6 \mathrm{ps})$, one $\mathrm{NO}_{2}$ molecule released and followed by one HONO released.

First, we examined the initial decomposition of $\beta$-HMX. Figure 2(a) shows a function of temperature of molecular fragments during the cook-off simulation. The system consists $8 \beta$-HMX molecules (224 atoms). When the temperature increases to $\sim 2200 \mathrm{~K}$ and instantaneous pressure increased to $\sim 1.0 \mathrm{GPa}$, the first reaction occurs, and two $\beta$-HMX molecules decomposed one by one almost at the same time, but leading to two distinct types of unimolecular decompositions, one releasing $\mathrm{HONO}$ and the other releasing $\mathrm{NO}_{2}$ molecule almost at the same time $\left(\mathrm{NO}_{2}\right.$ cleavage later than $\mathrm{HONO}$ release form another $\beta$-HMX molecule). Later after $2250 \mathrm{~K}$, there are more $\mathrm{NO}_{2}$ molecules released, see Figure 2(a). Thus, we consider that, for $\beta$-HMX, both unimolecular $\mathrm{HONO}$ releasing and $\mathrm{NO}_{2}$ cleavage reaction are the initial reactions. We will discuss the reaction 
mechanisms detailly below in the "Reaction mechanism from finite cluster calculations" section.

For the initial chemical reactions of $\delta$-HMX, the DFT-MD results is shown in Figure 2(b). Here $\delta$-HMX molecules start to react at $\sim 2010 \mathrm{~K}$, involving both $\mathrm{NO}_{2}$ cleavage and $\mathrm{HONO}$ releasing mechanisms. Different from the initial reactions of $\beta$-HMX, the $\mathrm{NO}_{2}$ molecule and the HONO molecule are released from the same HMX molecule. Thus, the initial reaction in $\delta$-HMX is different than for $\beta$-HMX case. Then $\sim 1$ ps later, at $2200 \mathrm{~K}$, HMX molecules decrease one by one, and more $\mathrm{NO}_{2}$ and $\mathrm{HONO}$ molecules releasing. This is similar to the RDX's initial reaction mechanism which the hydrogen-transition to form HONO occurs at the beginning 26-28.

\subsection{Reaction mechanism from finite cluster calculations}

To identify the initial thermal decomposition mechanism of $\beta$ - and $\delta$ - HMX detailly, we first extracted the activated intermediates, which involved in the first reactions, from the condensed phase DFT-MD simulation. Then we addressed the reaction mechanism as a gas phase reaction from finite cluster calculations as below.

\subsection{1. $\beta$-HMX structure transitions before initial decomposition.}

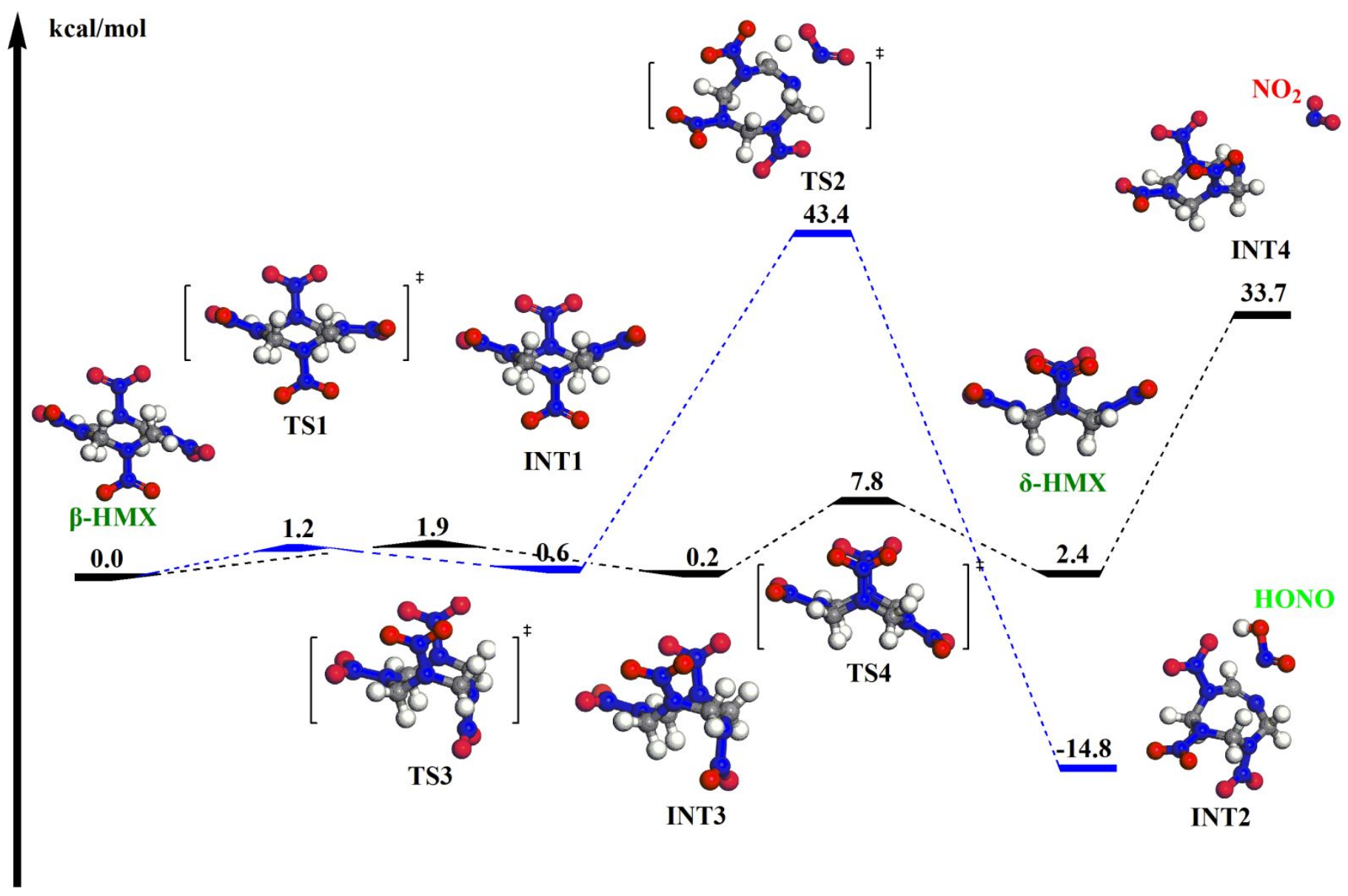

Figure 3. Mechanism of $\beta$-HMX structure transitions prior to initial decomposition. The gas phase calculations are performed here and start from the activated intermediates which extracted from the condensed phase DFT-MD trajectory (Configurations TS1 and TS3 were obtained at 1800-1900 K, and configurations INT2 and INT4 were obtained at 
2200 K. Configurations INT1, TS2, INT3 and TS4 are obtained from finite cluster DFT calculations.). This leads to release of one $\mathrm{NO}_{2}$ molecule and one $\mathrm{HONO}$ molecule. Unit is in $\mathrm{kcal} / \mathrm{mol}$.

First, we consider the $\beta$-HMX periodic system. As can be seen from Figure 2(a), at about $2200 \mathrm{~K}$, the two $\beta$-HMX molecules started to decompose simultaneously. We analyzed the DFT-MD trajectory to find that the first $\mathrm{HONO}$ releasing event and the $\mathrm{NO}_{2}$ cleavage event show two quite distinct kinds unimolecular decompositions $(\sim 1 / 2 \beta$-HMX molecules transfer to INT1 when the temperature increases to $1800 \mathrm{~K}$ before decomposition at $\sim 2200 \mathrm{~K}$ in DFT-MD simulation, see Figure S2). Here the simulated initial structure transition temperature is $1800 \mathrm{~K}$ as the decomposition temperature is $2200 \mathrm{~K}$ for $\beta$ - $\mathrm{HMX}$ (554 $\mathrm{K}$ in experiment), so the constant proportional transitions temperature is $\sim 453 \mathrm{~K}$, which is similar to the experimental results $(\sim 466 \mathrm{~K}){ }^{29}$. Then we separately optimized both two kinds of unimolecular decompositions as gas phase reactions. As described below for both cases, the nearby transition state (TS) were found with a single saddle point, and then we minimize them to find the nearby stable reactant and product species. In Figure 3, we addressed the observed reaction pathways and the free energy of each species and discussed below in detail.

We find (see Figure 3) the first HONO releasing unimolecular reaction pathway is $\boldsymbol{\beta}$-HMX - TS1 - INT1 -TS2 -INT2+HONO, which involves two steps. Starting with $\boldsymbol{\beta}$-HMX, the one of the parallel $\mathrm{N}-\mathrm{NO}_{2}$ groups (left and right $\mathrm{N}-\mathrm{NO}_{2}$ group in $\beta$-HMX) changes over time from a chair conformation to a boat conformation via TS1 (at $+1.2 \mathrm{kcal} / \mathrm{mol})$ to form INT1 $(+0.6 \mathrm{kcal} / \mathrm{mol}$ above $\boldsymbol{\beta}$-HMX). Then after formation of INT1 via the TS1 from $\boldsymbol{\beta}$-HMX, we find that one $\mathrm{H}$ atom near the parallel $\mathrm{NO}_{2}$ group move from $\mathrm{C}$ atom to the parallel $\mathrm{NO}_{2}$ group via TS2, then forms INT2 and release one HONO molecule. The barrier is $\Delta \mathrm{G}=+42.8 \mathrm{kcal} / \mathrm{mol}$, and this step is the rate-determining step (RDS). This is the first decomposition pathway we observed in the DFT-MD cookoff simulation (at $\sim 14 \mathrm{ps}$ ), Then 0.2 ps later, we find the first $\mathrm{NO}_{2}$ cleavage unimolecular decomposition process in the DFT-MD periodic system.

For the first $\mathrm{NO}_{2}$ cleavage unimolecular reaction, the pathway is $\boldsymbol{\beta}$-HMX - TS3 - INT3 -TS4 - $\boldsymbol{\delta}$-HMX - INT4+NO $\mathbf{O}_{2}$, which involves three steps, with $\beta$-HMX transforming to $\delta$-HMX before $\mathrm{NO}_{2}$ cleavage. Starting with $\boldsymbol{\beta}$-HMX, the first step involves one of the vertical $\mathrm{N}-\mathrm{NO}_{2}$ groups (up and down $\mathrm{N}-\mathrm{NO}_{2}$ group in $\beta$-HMX) changing over time from chair conformation to boat conformation via TS3 (at $+1.9 \mathrm{kcal} / \mathrm{mol}$ ) to form INT3 $\left(0.2 \mathrm{kcal} / \mathrm{mol}\right.$ above $\boldsymbol{\beta}$-HMX). The second step starts with the new formed vertical $\mathrm{N}-\mathrm{NO}_{2}$ group (right parallel $\mathrm{N}-\mathrm{NO}_{2}$ group in $\boldsymbol{\beta}$-HMX) over time from a vertical position to a parallel position to form a boat conformation via TS4 to form the intermediate, which is $\boldsymbol{\delta}-\mathbf{H M X}$. This reaction has a $+7.6 \mathrm{kcal} / \mathrm{mol}$ barrier. The third step to eliminate the $\mathrm{NO}_{2}$ molecule observed in the MD simulation involves breaking one parallel $\mathrm{N}-\mathrm{N}$ bond of $\boldsymbol{\delta}$ HMX to form INT4, which have a reaction energy of $31.3 \mathrm{kcal} / \mathrm{mol}$ above $\boldsymbol{\delta}$-HMX. The barrier of INT1 TS2 -INT2+HONO is $9.7 \mathrm{kcal} / \mathrm{mol}$ higher than the $\boldsymbol{\delta}$-HMX-INT4 reaction, but in the DFT-MD simulation HONO releasing and $\mathrm{NO}_{2}$ cleavage reaction are almost at the same time because in our cookoff simulations, 
we found that four molecules transform to INT1 molecular structure $(\sim 1800 \mathrm{~K})$ and while only one molecule transforms to full $\delta$-HMX $(\sim 2100 \mathrm{~K})$. and the HONO was observed from INT1 while the $\mathrm{NO}_{2}$ dissociation from $\delta$ - phase. This increase the probability of HONO releasing even the reaction barrier of HONO reaction is higher than $\mathrm{NO}_{2}$ dissociation. In addition, the fast quenching rate and small simulation cell also may accelerate the HONO reaction.

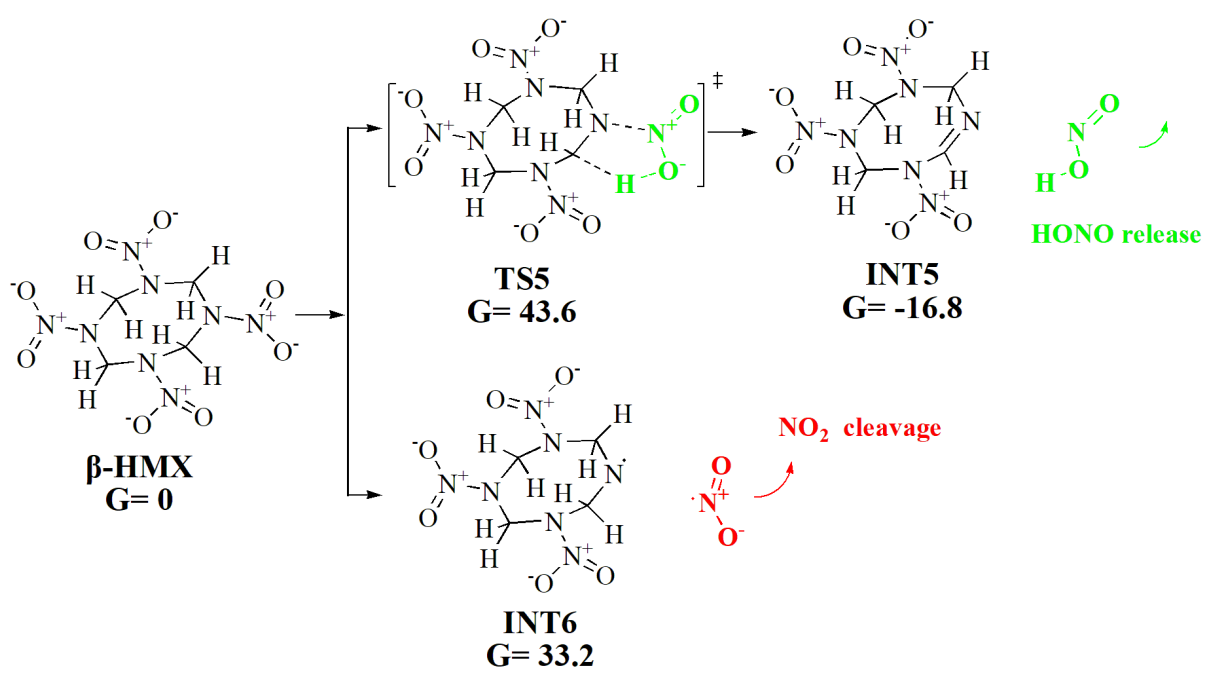

Figure 4. The mechanism of the proposed reaction pathway of $\mathrm{HONO}$ releasing and $\mathrm{NO}_{2}$ cleavage in $\beta$-HMX. The HONO releasing reaction has a barrier (TS5) of $43.6 \mathrm{kcal} / \mathrm{mol}$, and the $\mathrm{NO}_{2}$ cleavage reaction energy (barrier) is 33.2 $\mathrm{kcal} / \mathrm{mol}$ above $\boldsymbol{\beta}$-HMX. Units are in $\mathrm{kcal} / \mathrm{mol}$.

Although in the $\beta$-HMX periodic system, DFT-MD did not find a direct $\mathrm{HONO}$ or $\mathrm{NO}_{2}$ releasing reaction for at the first step, we also performed the adiabatic QM calculations and proposed one direct HONO releasing and one direct $\mathrm{NO}_{2}$ cleavage reaction (shown in Figure 4),. For the HONO releasing reaction, the $\beta$-HMX molecule stretches the N-N bond via TS5 (at $+43.6 \mathrm{kcal} / \mathrm{mol}$ ) to release a HONO molecule, leading to a barrier of $43.0 \mathrm{kcal} / \mathrm{mol}$ above INT1 (at $+0.6 \mathrm{kcal} / \mathrm{mol}$ ). For the $\mathrm{NO}_{2}$ releasing reaction, the $\beta$-HMX stretches the $\mathrm{N}$ $\mathrm{N}$ bond directly to INT6 which have a reaction energy of $33.2 \mathrm{kcal} / \mathrm{mol}$ above $\boldsymbol{\beta}$-HMX. As the barrier of $\boldsymbol{\beta}$ HMX - TS5 - INT5 is $0.2 \mathrm{kcal} / \mathrm{mol}$ higher than the INT1 - TS2 -INT2 reaction, and $\boldsymbol{\beta}$-HMX - INT6 is 1.9 $\mathrm{kcal} / \mathrm{mol}$ higher than the $\boldsymbol{\delta}$-HMX - INT4 reaction, this explains why we did not find this direct $\mathrm{NO}_{2}$ releasing reaction at the beginning decomposition.

\subsubsection{Unimolecular $\mathrm{NO}_{2}$ cleavage and $\mathrm{HONO}$ releasing reaction in $\delta$-HMX.}

Next, we consider the $\delta$-HMX case. Here the first reaction is an $\mathrm{N}-\mathrm{NO}_{2}$ cleavage and $\mathrm{HONO}$ releasing reaction in the same $\delta$-HMX molecule, leading to the unimolecular $\mathrm{NO}_{2}$ releasing and $\mathrm{HONO}$ releasing reaction, as shown in Figure 5. These two reactions take place in one $\delta$-HMX molecule in the condensed phase at about $2020 \mathrm{~K}$. At first, starting with $\boldsymbol{\delta}$-HMX, we find that the preferred pathway to release the $\mathrm{NO}_{2}$ molecule is stretching one vertical N-N bond to form INT7 (with a reaction energy of $31.6 \mathrm{kcal} / \mathrm{mol}$ above $\boldsymbol{\delta}$-HMX). Then from INT7, we find that one $\mathrm{H}$ atom near the parallel $\mathrm{NO}_{2}$ group moves from $\mathrm{C}$ atom to the nearby 
parallel $\mathrm{NO}_{2}$ group via TS6, then INT8 and one HONO molecule released, with a barrier of $\Delta \mathrm{G}=39.0$ $\mathrm{kcal} / \mathrm{mol}$ and release $-44.3 \mathrm{kcal} / \mathrm{mol}$ energy. In the gas phase calculation, we also investigate the other pathway to eliminate the HONO molecule from INT7 to form INT10 with $+49.0 \mathrm{kcal} / \mathrm{mol}$ barrier. This barrier is +10 $\mathrm{kcal} / \mathrm{mol}$ higher than the barriers of INT7 - TS6 - INT8 pathway, explaining why we do not observe it in the initial decomposition of $\delta$-HMX from DFT-MD simulations.

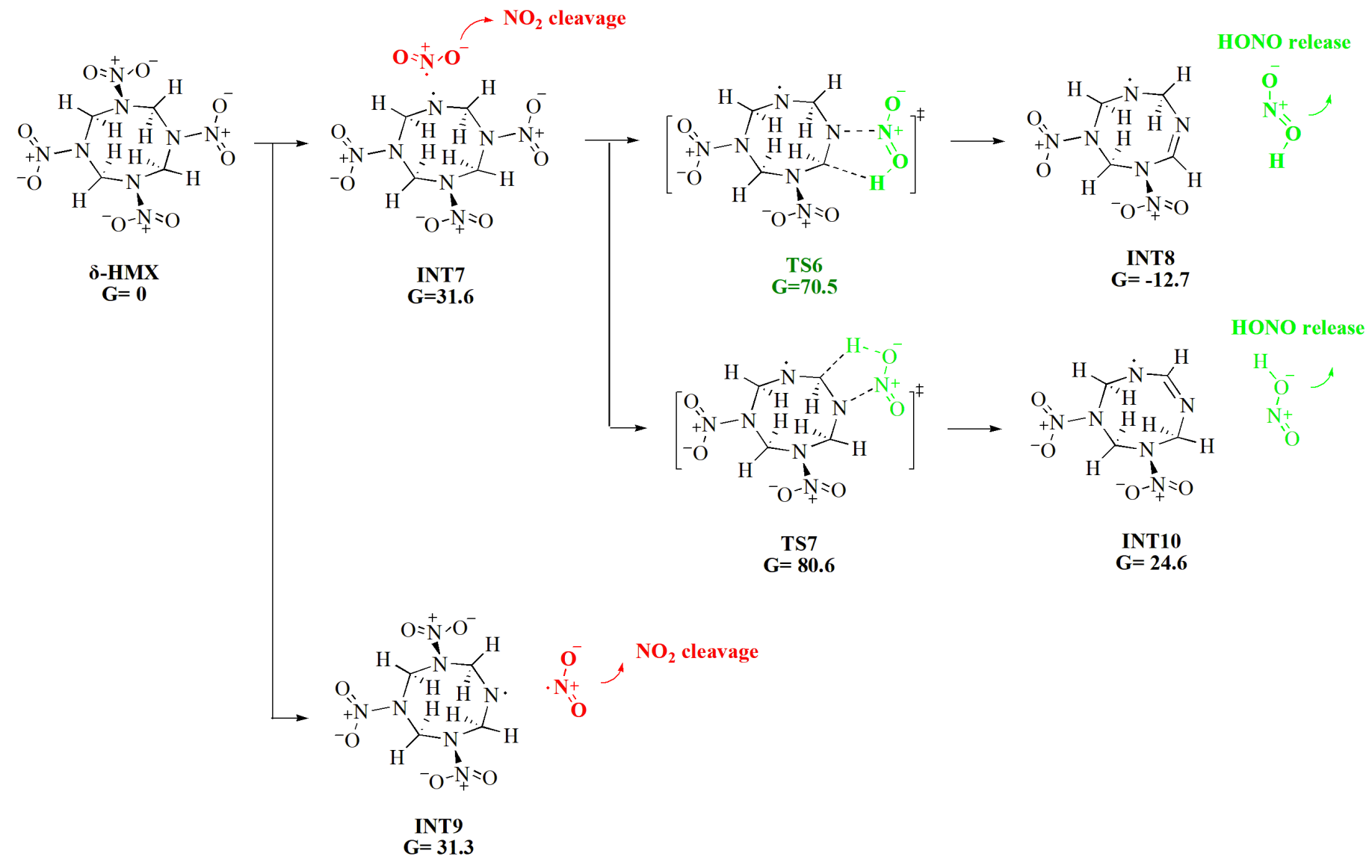

Figure 5 Mechanism of initial $\delta$-HMX unimolecular decomposition. The gas phase calculations are performed here and start from the activated intermediates which extracted from the condensed phase DFT-MD trajectory (Configurations INT7 were obtained at $\sim 2010 \mathrm{~K}$ and configurations INT8 were obtained at $\sim 2050 \mathrm{~K}$. Configurations TS6 and TS7 are obtained from finite cluster DFT calculations). This leads to release of one $\mathrm{NO}_{2}$ molecule and one $\mathrm{HONO}$ molecule. Configurations INT9 is the pathway to eliminate the $\mathrm{NO}_{2}$ molecule breaking one parallel N-N bond from $\delta$-HMX is not observed in the initial decomposition of $\delta$-HMX from DFT-MD simulations but obtained from finite cluster DFT calculations. Unit is in $\mathrm{kcal} / \mathrm{mol}$.

\section{CONCLSUION}

The goal of this work was to identify the initial reaction mechanism of $\beta$ - and $\delta$ - HMX decomposes to find the events in this well-used EM, as it evolves to different structures transitions before decomposition that followed different reaction pathways to eventually form the different final products observed theoretically and experimentally. Indeed, from our DFT-MD simulations, we obtained a detailed, molecular-level reaction 
pathway of the thermal decomposition in condensed phase $\beta$ - and $\delta$ - HMX. This enables information about the complex physics or chemistry involved to be extracted, including structure transition, single versus multiple steps reaction sequences. Key points of our simulations are

(1) There are two different initial unimolecular reaction pathways in $\beta$-HMX, both operating simultaneously. One releases the first HONO while the other releases the first $\mathrm{NO}_{2}$ molecules.

(2) For the HONO releasing reaction, $\beta$-HMX first transforms the structure to an intermediate in which one of the parallel $\mathrm{N}-\mathrm{NO}_{2}$ groups changes over tune from chair to boat conformations, with a low barrier of $+1.2 \mathrm{kcal} / \mathrm{mol}$, suggesting that this reaction may proceed very easily before decomposition reaction. This is followed by unimolecular HONO release $(42.8 \mathrm{kcal} / \mathrm{mol}$ barrier, RDS).

(3) For the $\mathrm{NO}_{2}$ cleavage reaction, $\beta$-HMX first transforms to the $\delta$-HMX structure via INT3 in which one of the vertical $\mathrm{N}-\mathrm{NO}_{2}$ groups changes over time from chair to boat conformations in two steps, with low barriers of +1.9 and $+7.6 \mathrm{kcal} / \mathrm{mol}$ for each step, followed by unimolecular $\mathrm{NO}_{2}$ release (+31.3 kcal/mol barrier).

(4) $\delta$-HMX initially performs a both unimolecular $\mathrm{NO}_{2}$ cleavage and $\mathrm{HONO}$ releasing reactions in one $\delta$-HMX molecule, with the barriers of $+31.6\left(\mathrm{NO}_{2}\right.$ cleavage) and $+38.9 \mathrm{kcal} / \mathrm{mol}$ (HONO releasing) for each step.

These simulations addressed and rationalized the decomposition mechanisms of $\beta$-HMX, showing that the formation of $\delta$-HMX occurs first, as experimental studies had previously speculated. We expect these theoretical studies of the initial reaction of $\beta$ - and $\delta$ - HMX might stimulate further experimental mechanisms studies of HMX, particularly the ignition, and characterization.

\section{ASSOCIATED CONTENT}

\section{Supporting Information}

Unit cell information and initial simulation structures of $\beta$-HMX and $\delta$-HMX, the bond type and bond cutoff value which used in the fragment analysis, atomic coordinates of all intermediates and TS shown in this study. These materials are available free of charge via the Internet at http://pubs.acs.org.

\section{AUTHOR INFORMATION}

\section{Corresponding Author}

*E-mail: yecc@sustech.edu.cn; wag@ @ caltech.edu

\section{Notes}

The authors declare no competing financial interest. 


\section{ACKNOWLEDGMENTS}

This research was supported and funded by ONR (N00014-12-1-0538 and N00014-16-1-2059). W. Zhang and C. Ye acknowledges the support program from Guangdong Innovation Research Team Project (Grant No. 2017ZT07C062), and Shenzhen Pengcheng-Scholarship program.

\section{REFERENCES}

1. Henson, B. F.; Asay, B. W.; Sander, R. K.; Son, S. F.; Robinson, J. M.; Dickson, P. M., Dynamic measurement of the HMX beta-delta phase transition by second harmonic generation. Phys. Rev. Lett. 1999, 82 (6), 1213-1216.

2. Brill, T. B.; Reese, C. O., Analysis of Intra- and Intermolecular Interactions Relating to the Thermophyslcal Behavior of $\alpha$-, $\beta$-, and $\delta$-Octahydro-1,3,5,7-tetranltro-1,3,5,7-tetraazocIne. J. Phys. Chem. 1980, 84, 1376-1380.

3. Cady, H. H.; Stmith, L. C. Studies on the polymorphs of HMX; LAMS-2652; 1962.

4. Asay, B. W.; Henson, B. F.; Smilowitz, L. A. B.; Dickson, P. M., On the difference in impact sensitivity of beta and delta HMX. J. Energ. Mater. 2003, 21 (4), 223-235.

5. Brill, T. B.; Karpowicz, R. J., Solid-Phase Transition Kinetics - the Role of Intermolecular Forces in the CondensedPhase Decomposition of Octahydro-1,3,5,7-Tetranitro-1,3,5,7-Tetrazocine. J. Phys. Chem. 1982, 86 (21), 4260-4265.

6. Henson, B. F.; Smilowitz, L.; Asay, B. W.; Dickson, P. M., The beta-delta phase transition in the energetic nitramine octahydro-1,3,5,7-tetranitro-1,3,5,7-tetrazocine: Thermodynamics. J. Chem. Phys. 2002, 117 (8), 3780-3788.

7. Levitas, V. I.; Smilowitz, L. B.; Henson, B. F.; Asay, B. W., Interfacial and volumetric kinetics of the beta to delta phase transition in the energetic nitramine octahydro-1,3,5,7-tetranitro-1,3,5,7-tetrazocine based on the virtual melting mechanism. J. Chem. Phys. 2006, 124 (2), 026101.

8. Smilowitz, L.; Henson, B. F.; Greenfield, M.; Sas, A.; Asay, B. W.; Dickson, P. M., On the nucleation mechanism of the beta-delta phase transition in the energetic nitramine octahydro-1,3,5,7-tetranitro-1,3,5,7-tetrazocine. J. Chem. Phys. 2004, 121 (11), 5550-5552.

9. Smilowitz, L.; Henson, B. F.; Asay, B. W.; Dickson, P. M., The beta-delta phase transition in the energetic nitramineoctahydro-1,3,5,7-tetranitro-1,3,5,7-tetrazocine: Kinetics. J. Chem. Phys. 2002, 117 (8), 3789-3798.

10. Furman, D.; Kosloff, R.; Dubnikova, F.; Zybin, S. V.; Goddard III, W. A.; Rom, N.; Hirshberg, B.; Zeiri, Y., Decomposition of Condensed Phase Energetic Materials: Interplay between Uni- and Bimolecular Mechanisms. J. Am. Chem. Soc. 2014, 136 (11), 4192-4200.

11. An, Q.; Liu, W.; Goddard III, W. A.; Cheng, T.; Zybin, S. V.; Xiao, H., Initial Steps of Thermal Decomposition of Dihydroxylammonium 5,5'-bistetrazole-1,1'-diolate Crystals from Quantum Mechanics. J. Phys. Chem. C 2014, 118 (46), 27175-27181.

12. Ye, C. C.; An, Q.; Goddard, W. A.; Cheng, T.; Zybin, S.; Ju, X. H., Initial Decomposition Reactions of Bicyclo-HMX [BCHMX orcis-1,3,4,6-Tetranitrooctahydroimidazo-[4,5-d]imidazole] from Quantum Molecular Dynamics Simulations. $J$. Phys. Chem. C 2015, 119, 2290-2296.

13. Ye, C. C.; An, Q.; Goddard III, W. A.; Cheng, T.; Liu, W. G.; Zybin, S. V.; Ju, X. H., Initial decomposition reaction of di-tetrazine-tetroxide (DTTO) from quantum molecular dynamics: implications for a promising energetic material. J. Mater. Chem. A 2015, 3 (5), 1972-1978.

14. Ye, C. C.; An, Q.; Cheng, T.; Zybin, S.; Naserifar, S.; Ju, X. H.; Goddard III, W. A., Reaction mechanism from quantum molecular dynamics for the initial thermal decomposition of 2,4,6-triamino-1,3,5-triazine-1,3,5-trioxide (MTO) and 2,4,6trinitro-1,3,5-triazine-1,3,5-trioxide (MTO3N), promising green energetic materials. J. Mater. Chem. A 2015, 3 (22), 1204412050 .

15. Guo, D.; An, Q., Thermal Stability and Detonation Properties of Potassium 4,4'-Bis(dinitromethyl)-3,3'-Azofurazanate, an Environmental Friendly Energetic Three-Dimensional Metal-Organic Framework. ACS Appl. Mater. Interfaces 2019, 11(1), 1512-1519.

16. Kim, H.; Choi, J. M.; Goddard III, W. A., Universal Correction of Density Functional Theory to Include London 
Dispersion (up to Lr, Element 103). J. Phys. Chem. Lett. 2012, 3 (3), 360-363.

17. Kohn, W.; Sham, L. J., Self-Consistent Equations Including Exchange and Correlation Effects. Phys. Rev. 1965, 140 (4A), 1133-1138.

18. Hohenberg, P.; Kohn, W., Inhomogeneous Electron Gas. Phys. Rev. 1964, 136 (3B), B864-B871.

19. Kresse, G., Ab-Initio Molecular-Dynamics for Liquid-Metals. J. Non-Cryst Solids 1995, 193, $222-229$.

20. Kresse, G.; Furthmuller, J., Efficiency of ab-initio total energy calculations for metals and semiconductors using a planewave basis set. Comp. Mater. Sci. 1996, 6 (1), 15-50.

21. Kresse, G.; Furthmuller, J., Efficient iterative schemes for ab initio total-energy calculations using a plane-wave basis set. Phys. Rev. B 1996, 54 (16), 11169-11186.

22. Kresse, G.; Joubert, D., From ultrasoft pseudopotentials to the projector augmented-wave method. Phys. Rev. B 1999, 59 (3), 1758-1775.

23. Bochevarov, A. D.; Harder, E.; Hughes, T. F.; Greenwood, J. R.; Braden, D. A.; Philipp, D. M.; Rinaldo, D.; Halls, M. D.; Zhang, J.; Friesner, R. A., Jaguar: A high-performance quantum chemistry software program with strengths in life and materials sciences. Int. J. Quantum Chem. 2013, 113 (18), 2110-2142.

24. Fukui, K., The Path of Chemical-Reactions - the Irc Approach. Accounts Chem. Res. 1981, 14 (12), $363-368$.

25. Han, S. P.; van Duin, A. C.; Goddard III, W. A.; Strachan, A., Thermal decomposition of condensed-phase nitromethane from molecular dynamics from ReaxFF reactive dynamics. J. Phys. Chem. B 2011, 115 (20), 6534-40.

26. Minier, L. M.; Brower, K. R.; Oxley, J. C., Role of Intermolecular Reactions in Thermolysis of Aromatic NitroCompounds in Supercritical Aromatic Solvents. J. Org. Chem. 1991, 56 (10), 3306-3314.

27. Davis, L. L.; Brower, K. R., Reactions of organic compounds in explosive-driven shock waves. J. Phys. Chem. 1996, 100 (48), 18775-18783.

28. Chakraborty, D.; Muller, R. P.; Dasgupta, S.; Goddard III, W. A., The mechanism for unimolecular decomposition of RDX (1,3,5-trinitro-1,3,5-triazine), an ab initio study. J. Phys. Chem. A 2000, 104 (11), 2261-2272.

29. Tang, Z.; Yang, L.; Qiao, X. J.; Zhang, T. L.; Yu, W. F., On thermal decomposition kinetics and thermal safety of HMX. Chin. J. Energ. mater. 2011, 19 (4), 396-400. 


\section{TOC Graphic}

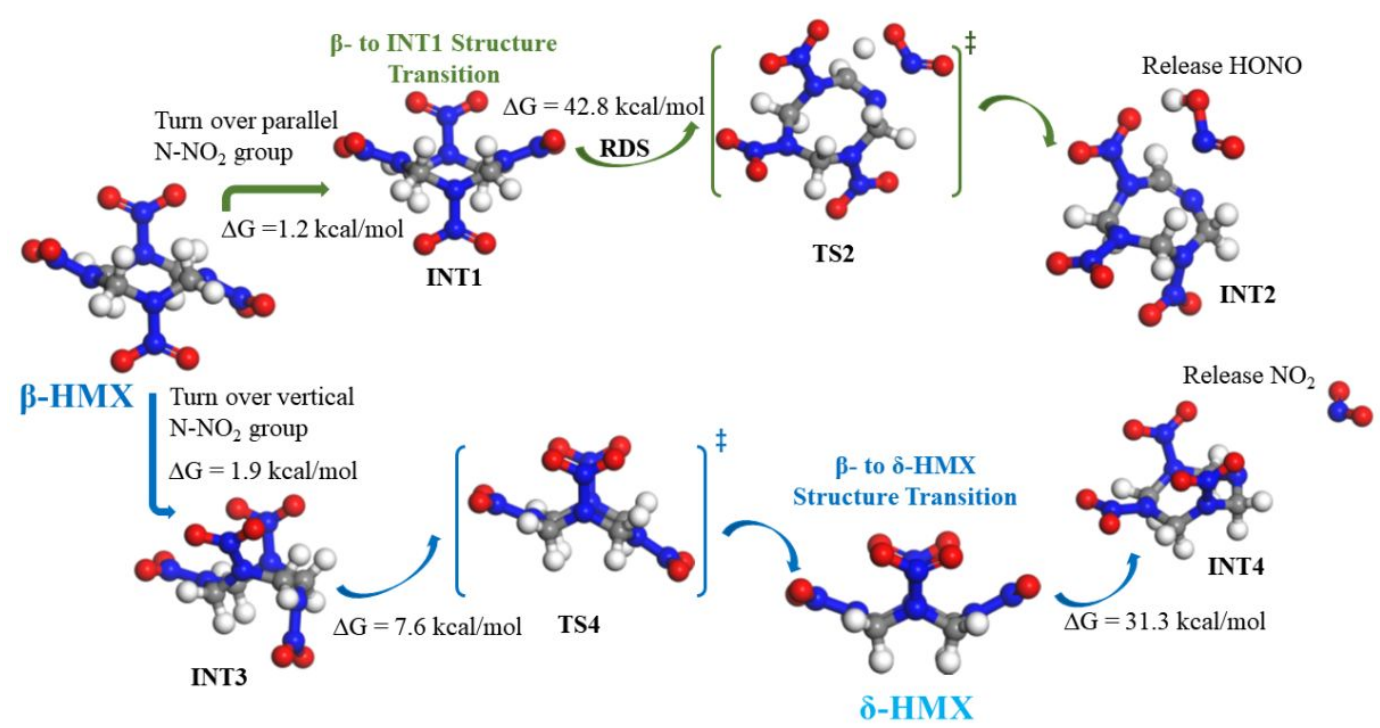

